1 Högberg, L., and Vogel, K., Nucl. Instr. Meth., 10, 95 (1960).

2 Wooding, E. R., Nature, 199, 272 (1963).

3 Johnson, P. O., Amer. J. Phys., 33, 119 (1965).

4 Rayle, W. D., NASA Tech. Note, TND 3188 (1966).

${ }^{5}$ Nilsson, N. R., Steinvall, O., Subramanian, C. K., and Högberg, L., Phys. Script., 1, 153 (1970).

6 Stamper, J. A., Papadopoulos, K., and Sodan, R. N., Phys. Rev. Lett., 26, 1012 (1971).

${ }^{7}$ Floux, F., Cognard, D., Saleres, A., and Bobin, J. L., Digest of Papers, Seventh Intern. Quantum Electronics Conf. Montreal, May 1972 (IEEE, 1972).

8 Viemester, F. E., The Lightning Book, plate 36 (Doubleday and Co. Inc., New York, 1961).

\section{Antimatter and Ball Lightning}

Ashby and Whitehead ${ }^{1}$ have published evidence of the observation of fluxes of photons lasting $\leq 1 \mathrm{~s}$ at an energy corresponding to positron annihilation, in connexion with the possibility that "ball lightning" may be associated with the existence in the terrestrial environment of small quantities $\left(\sim 10^{-11} \mathrm{~g}\right)$ of antimatter. Here I shall present evidence that fluxes of this type, at about the observed frequency, can be expected from extensive air showers caused by primary cosmic rays of energy $E_{0} \sim 10^{16} \mathrm{eV}$.

It can be shown ${ }^{2}$ that more than $90 \%$ of the energy in an air shower caused by a primary in this energy range appears in the form of superimposed electromagnetic showers from photons produced in $\pi^{\circ}$ decay. Further, recent Monte Carlo studies at this laboratory by $\mathbf{R}$. L. Ford, using a program originally due to $\mathbf{H}$. H. Nagel, show that in electromagnetic showers at $10^{9} \mathrm{eV}$ and $8 \times 10^{9} \mathrm{eV}$, about seventy-five positrons are produced for each $10^{9} \mathrm{eV}$ of primary energy. (These results are for lead, but will presumably not differ significantly for other materials.) As these positrons will in general be fast, only about $70 \%$ of them will annihilate at rest $^{3}$, and I shall neglect the remainder. I therefore assume here that about $5.5 \%$ of the primary cosmic ray energy appears in the form of photons of energy $511 \mathrm{keV}$.

How would this radiation be distributed in space? Hayakawa $^{4}$ gives the radius of the core of such a shower, from which an effective value of $2 \mathrm{~m}$ can be taken. Further, when the core hits the ground, it enters a medium where the radiation length (in $\mathrm{m}$ ) is some three orders of magnitude shorter. Hence, for the first few radiation lengths in the ground, its longitudinal development will be typical of a solid whereas its lateral size will remain characteristic of the air. The annihilation of slow positrons will cause the isotropic emission of $511 \mathrm{keV}$ photons. Because of the absorption in the ground, and solid angle effects, I shall consider only the first radiation length $(\sim 20 \mathrm{~cm})$ of the ground. Thus a vertical shower has an approximately disk shaped source of radius $r=2 \mathrm{~m}$, thickness $\sim 20 \mathrm{~cm}$. A spherical detector of radius $\delta$ at a height $h$ above the ground will then collect a fraction

$$
\frac{\delta^{2}}{4 r^{2}} \log _{e}\left(1+\frac{r^{2}}{h^{2}}\right)
$$

of the emitted photons. If $\delta=6 \mathrm{~cm}, r=2 \mathrm{~m}$, and $h=1 \mathrm{~m}$, this fraction is $3.6 \times 10^{-4}$.

I shall now consider the number $N$ of photons emitted by the disk. On page 472 of ref. 4 Hayakawa gives the longitudinal development of a shower, and using this, the relative energy deposition can be approximated for simplicity by a function which rises linearly from zero to a maximum at a depth $l$ and then declines linearly to zero at a total depth $3 l$, where $l$ is the depth of the "shower maximum" for an electromagnetic shower of the same initial energy $E_{0}$. It can then be shown that the expected energy deposition at an atmospheric depth $d$ will be

$$
E^{\prime}=E_{0} \frac{(3 l-d)}{3 l^{2}}
$$

per radiation length, over the range $l<d<3 l$. The number of $511 \mathrm{keV}$ photons will then be $N=0.055 E^{\prime} / m_{\mathrm{e}}$. The condition that $d<3 l$ defines a solid angle for acceptance

$$
\Omega=2 \pi\left(1-\frac{d}{3 l}\right)
$$

It turns out that

$$
\bar{E}^{\prime}=E_{0}\left[\frac{1}{l}-\frac{d}{l(3 l-d)} \log _{\mathrm{e}} \frac{3 l}{d}\right]=E_{0} f
$$

As $d=28$ radiation lengths of air at sea level, and as $l$ varies with $\log E_{0}$, it follows that the factor $f$ is almost constant at $\sim 1 / 60$ for energies above $\sim 10^{15} \mathrm{eV}$ (for which $l=16$ radiation lengths).

The average flux of the four events seen by Ashby and Whitehead is 8,200 counts. This gives

$$
\begin{aligned}
\bar{N}=\frac{8,200}{3.6 \times 10^{-4}} & =2.27 \times 10^{7}, \\
\vec{E}^{\prime} & =\frac{m_{\mathrm{e}}}{0.055} \times 2.27 \times 10^{7}=2.1 \times 10^{14} \mathrm{eV}
\end{aligned}
$$

so that an initial energy $E_{0}$ of $1.25 \times 10^{16} \mathrm{eV}$ is required. Here $l=18.5$ radiation lengths, so that $\Omega=3.1 \mathrm{sr}$. The equipment was active for $\sim 1.3 \times 10^{7} \mathrm{~s}$, and if a "sensitive area" of $\sim 10 \mathrm{~m}^{2}$ is assumed a total exposure of $1.3 \times 10^{7} \times 3.1 \times 10 \times 10^{4}=4.04 \times$ $10^{12} \mathrm{~cm}^{2} \mathrm{sr} \mathrm{s}$ is indicated. Hayakawa (ref. 4, page 569) gives the primary flux at $1.25 \times 10^{16} \mathrm{eV}$ as $0.6 \times 10^{-12}\left(\mathrm{~cm}^{2} \mathrm{~s} \mathrm{sr}\right)^{-1}$, so that about 2.5 events are to be expected-almost the number seen by Ashby and Whitehead.

These arguments are by no means precise, but they do indicate that substantial fluxes of $511 \mathrm{keV}$ photons are to be expected in the cores of extensive air showers. Quite apart from any relevance to the question of ball lightning, this phenomenon might be of use in cosmic ray studies.

\section{J. F. Crawford}

W. W. Hansen Laboratories of Physics,

Stanford University,

Stanford,

California 94305

Received February 18, 1972.

1 Ashby, D. E. T. F., and Whitehead, C., Nature, 230, 180 (1971).

2 Murzin, V. S., Prog. in Elem. Part. and Cosmic Ray Physics, 9, 250 (1967).

3 Bethe, H. A., Proc. Roy. Soc., A, 150, 129 (1935).

4 Hayakawa, S., Cosmic Ray Physics (Wiley Interscience, 1969).

\section{BIOLOGICAL SCIENCES}

\section{Enzymatic Cleavage of Methotrexate provides a Method for Prevention of Drug Toxicity}

Methotrexate, a widely used and effective antineoplastic agent, is eliminated primarily by urinary excretion in man ${ }^{1}$. In species such as the rabbit, which possess hepatic enzymes capable of metabolizing methotrexate to inactive products, the drug is rapidly degraded and virtually non-toxic ${ }^{2}$. In species which do not metabolize the drug, such as mouse and man, the dose-limiting bone marrow and gastro-intestinal toxicity seems to result from persistence of low $\left(10^{-7}\right.$ to $\left.10^{-9} \mathrm{M}\right)$ plasma levels of methotrexate for many hours after its administration $^{3,4}$. To counteract this toxicity, 5-formyl tetrahydrofolate, or leucovorin, has been given 12-36 h after high doses of methotrexate, allowing an improvement in the drug's therapeutic index ${ }^{5,6}$. 